\title{
Ecologic association between influenza and COVID-19 mortality rates in European countries
}

\section{Original Paper}

Cite this article: Petti S, Cowling BJ (2020). Ecologic association between influenza and COVID-19 mortality rates in European countries. Epidemiology and Infection 148, e209, 1-7. https://doi.org/10.1017/ S0950268820002125

Received: 7 June 2020

Revised: 12 August 2020

Accepted: 8 September 2020

Key words:

COVID-19; influenza

Author for correspondence:

S. Petti, E-mail: stefano.petti@uniroma1.it (c) The Author(s), 2020. Published by Cambridge University Press. This is an Open Access article, distributed under the terms of the Creative Commons Attribution licence (http://creativecommons.org/licenses/by/4.0/), which permits unrestricted re-use, distribution, and reproduction in any medium, provided the original work is properly cited.

\section{CAMBRIDGE} UNIVERSITY PRESS

\section{S. Petti ${ }^{1}(1)$ and B. J. Cowling ${ }^{2}(1)$}

${ }^{1}$ Department of Public Health and Infectious Diseases, Sapienza University, Rome, Italy and ${ }^{2}$ WHO Collaborating Centre for Infectious Disease Epidemiology and Control, School of Public Health, Li Ka Shing Faculty of Medicine, The University of Hong Kong, Hong Kong, China

\begin{abstract}
Ecologic studies investigating COVID-19 mortality determinants, used to make predictions and design public health control measures, generally focused on population-based variable counterparts of individual-based risk factors. Influenza is not causally associated with COVID-19, but shares population-based determinants, such as similar incidence/mortality trends, transmission patterns, efficacy of non-pharmaceutical interventions, comorbidities and underdiagnosis. We investigated the ecologic association between influenza mortality rates and COVID-19 mortality rates in the European context. We considered the 3-year average influenza (2014-2016) and COVID-19 (31 May 2020) crude mortality rates in 34 countries using EUROSTAT and ECDC databases and performed correlation and regression analyses. The two variables - log transformed, showed significant Spearman's correlation $\rho=0.439(P=0.01)$, and regression coefficients, $b=0.743$ (95\% confidence interval, $0.272-$ $1.214 ; R^{2}=0.244 ; P=0.003$ ), $b=0.472$ (95\% confidence interval, $0.067-0.878 ; R^{2}=0.549$; $P=0.02$ ), unadjusted and adjusted for confounders (population size and cardiovascular disease mortality), respectively. Common significant determinants of both COVID-19 and influenza mortality rates were life expectancy, influenza vaccination in the elderly (direct associations), number of hospital beds per population unit and crude cardiovascular disease mortality rate (inverse associations). This analysis suggests that influenza mortality rates were independently associated with COVID-19 mortality rates in Europe, with implications for public health preparedness, and implies preliminary undetected SARS-CoV-2 spread in Europe.
\end{abstract}

\section{Introduction}

The COVID-19 outbreak, caused by the severe acute respiratory syndrome coronavirus 2, SARS-CoV-2, evolved in two distinct phases. The former was a local outbreak first detected in China in December 2019, and the latter the subsequent spread of the virus to the rest of the world. On 11 March 2020, the World Health Organization (WHO) made the assessment that the COVID-19 outbreak could be characterised as a pandemic [1]. In the beginning of the second phase, the COVID-19 pandemic has been particularly severe in Europe and North America. Indeed, by the end of May mortality rates were as high as 22.5 and 14.3 per 100000 , respectively, while in the remaining continents rates were lower than 1.0 per 100000 [2]. COVID-19 spread in Europe has been uneven, with Italy experiencing the highest death toll in February and March, followed by other Western countries. By the end of May, COVID-19 mortality rates were ranging between higher than 50 per 100000 in Belgium, Spain, UK and Italy, and lower than 2 per 100000 in Slovakia, Greece and Bulgaria. Several factors probably explained these varying mortality rates, such as nature and timeliness of implementation of COVID-19 control policy measures [3], demographic variables [4], healthcare system quality and ability to manage the rapid COVID-19 spread [5-7] and the timing of SARS-CoV-2 introduction in the community [8].

As for the last issue, several studies suggest that SARS-CoV-2 could be circulating in Europe before the detection of the early COVID-19 cases. Namely, although the first Italian patient with COVID-19 was identified on 20 February 2020 and the earlier containment measures were already implemented on 21 February [9], on 21-29 February almost 3\% of the residents of a small Italian town in the area of the outbreak epicentre resulted infected with SARS-CoV-2 [10]. In addition, seroprevalence of SARS-CoV-2 (test accuracy, 100\% sensitivity, 98.2\% specificity) in blood samples collected in December 2019 from healthy donors living in Milan was as high as $2.7 \%$, according to a preprint survey [11], while a nasopharyngeal swab collected in December 2019 from a French patient admitted to intensive care unit for severe influenza-like illness (ILI) was reanalysed in April 2020 and resulted positive for SARS-CoV-2 [12]. Phylogenetic analyses also support this hypothesis, showing that SARS-CoV-2 was circulating outside China since fall 2019, and there have been multiple SARS-CoV-2 introductions in Europe [13-15]. Uncontrolled virus circulation in humans before its discovery is typical of 
the human coronavirus (HCoV) species [16]. The investigation of the dynamics of the COVID-19 outbreak must, therefore, account for SARS-CoV-2 circulation that occurred before the implementation of nationwide public health measures and that could help explain why SARS-CoV-2 was ubiquitous in Europe as early as in May, with seroprevalence estimates of $1-2 \%$ in blood donors and $4-5 \%$ in the general population, with peaks of $10-15 \%$ [17-19], that were likely limited by the unprecedented public health measures implemented in most countries after the detection of COVID-19 cases [3].

The design of specific anti-COVID-19 control measures, the implementation of community-based control strategies and the proper allocation of resources, can benefit from the investigation of the country-based determinants associated with COVID-19 mortality and severity. For this reason, during the SARS-CoV-2 pandemic many ecologic studies have been performed. The majority of them considered variables that reflected at population level the risk factors for COVID-19 severity at an individual level, such as population ageing, prevalence of diseases associated with COVID-19 death and severity, healthcare system capacity to face the public health emergency, etc. [4, 20-22]. The assessment of COVID-19 mortality determinants could benefit from similarities between this and other respiratory infectious diseases, particularly influenza, as these diseases share several characteristics. Indeed, during the 2019-2020 season, ILI and COVID-19-like illness (CLI) followed similar weekly incidence rate trends, although absolute values were different, as shown by the National Syndromic Surveillance Program in USA [23], and the general practitioners' network in France [24]. In addition, the public health measures taken to constrain the SARS-CoV-2 outbreak in Japan also limited the activity of seasonal influenza [25]. Similarity between influenza and COVID-19 incidence and mortality rates, however, does not result in the equivalence between these diseases, since influenza virus and SARS-CoV-2 are two distinct species of enveloped RNA virus belonging to two different families. Indeed, there are clinical, epidemiological and biological differences between the two diseases [26] which lead to differences in disease burden, case-fatality rates, proportion of asymptomatic individuals, etc. However, Tolksdorf and colleagues found that community-based influenza determinants could somewhat predict COVID-19 burden [27]. Thus, in addition to the aforementioned aggregated variables, population-level counterparts of individual-level COVID-19 severity risk factors, influenza-related variables could be eligible as determinant of COVID-19 mortality in European countries, to build an accurate COVID-19 mortality model.

Therefore, the aim of this study was to investigate the ecologic association between influenza and COVID-19 mortality rates in the European countries.

\section{Methods}

\section{Data sources and parameters}

Data on COVID-19 deaths in 34 European countries were gathered from the COVID-19 database of the European Centre for Disease Prevention and Control (ECDC) [2]. Crude COVID-19 mortality rates (number of deaths per 100000 ) were assessed using the population on 1 January 2020 extracted from the EUROSTAT database [28]. This study focused on the first major epidemic waves of COVID-19 in Europe up until 31 May 2020 [29].
Crude influenza mortality rates for the last available years (i.e. 2014-2016), were assessed. The number of influenza deaths in a given year and the population on 1 January of that year were extracted from the EUROSTAT database [28] and crude mortality rates calculated. Then, for each country the 3-year average influenza mortality rates were assessed.

Some, but not all demographic, health and healthcare determinants, potentially associated with influenza and COVID-19 mortality rates also were extracted through the same database and assessed. Namely, 3-year average population (2014-2016), life expectancy at birth (2018), healthy life years at birth (2018, available only for a subset of countries), 3-year average crude all-cause mortality rate (per 1000, 2014-2016), 3-year average crude pneumonia mortality rate (per $100000,2014-2016$ ), crude cardiovascular disease (CVD) mortality rate (per 100000,2016 ), number of hospital beds per 100000 (2017), 3-year average influenza vaccination coverage in population aged $\geq 65$ years (2014-2016, available only for a subset of countries).

\section{Data analysis}

The association between COVID-19 and 3-year average influenza mortality rates was explored using the nonparametric Spearman's correlation coefficient $\rho$. Then, the COVID-19 and 3-year average influenza mortality rates were log transformed to normalise variances and simple and multiple regression analyses were performed with log COVID-19 mortality rate as dependent variable. Zero values that could not be log transformed were given the lowest detected value.

The explanatory variables initially considered for the multiple regression analysis were, 3-year average population, life expectancy at birth, healthy life years at birth, 3-year average influenza vaccination coverage in population aged $\geq 65$ years, 3 -year average crude all-cause mortality rate, 3 -year average crude pneumonia mortality rate, crude CVD mortality rate, number of hospital beds per population unit. Mortality rates, average population and number of hospital beds were log transformed. Correlation matrix was preliminarily performed to investigate collinearity that could inflate the coefficient estimates. Only non-correlated variables, with Pearson's correlation coefficients $<0.4$, were considered. Influenza mortality was forced into the model. In order to control the regression model for overfitting, due to the inclusion of unneeded predictors, the regression was initially run with all the non-collinear variables and variables that yielded statistically non-significant coefficient estimates $(P \geq 0.05)$ were excluded, thus obtaining a limited set of meaningful variables.

In order to study whether influenza and COVID-19 mortality rates shared common determinants that may help justify the similarity between these population-based variables, a series of simple regression analyses was designed treating both COVID-19 and influenza mortality rates as dependent variables, and using the same set of determinants considered for the multiple regression analysis.

Since the influenza mortality rates could be unreliable in small countries, the analysis was repeated considering only countries with population higher than 2000000 individuals.

The agreement between influenza and COVID-19 mortality severity also was studied. More specifically, countries were grouped in quartiles according to the two mortality rates, and the agreement between influenza and COVID-19 quartiles was investigated. The absolute agreement (i.e. the proportion of countries located in the same influenza and COVID-19 quartile), and 
the intraclass correlation coefficient (ICC), were assessed. Two-way absolute agreement single measure ICC was used, considering the country classification into COVID-19 mortality quartiles as reference value [30].

In order to explore the potential of a multivariate ecologic study to predict COVID-19 mortality (actually, this was not an aim of the current study), the agreement between the observed COVID-19 mortality and the COVID-19 mortality estimated by the multiple regression analysis, also was investigated using the same methodology.

\section{Results}

There were 34 countries included in the analysis (Supplementary Table S1). The overall COVID-19 mortality rate was 27.76 per 100000 , the lowest and highest rates were reported in Slovakia and Belgium with 0.51 and 82.52 per 100000 , respectively. The overall 3-year average influenza mortality rate in the 34 countries was roughly 30 times lower, namely, 0.91 per 100000 , and the lowest and highest rates were reported in Lichtenstein and Finland, with 0.00 and 2.49 per 100000 , respectively. The two mortality rates were correlated (Spearman's $\rho=0.439 ; P=0.01$ ).

The simple regression coefficient was $b=0.743$ (95\% confidence interval, $0.272-1.214 ; \quad P=0.003)$, with $R^{2}=0.244$ (Table 1), suggesting that 3 -year average influenza mortality rate could explain $24.4 \%$ of the between-country variations in COVID-19 mortality rate.

Several investigated determinants were highly inter-correlated (Supplementary Table S2), and after the elimination of collinear variables, four variables were remaining that were considered for the initial multiple regression model (Table 1). After the removal of pneumonia mortality rate, the regression coefficient for influenza mortality resulting from the final model was $b=$ 0.472 (95\% confidence interval, 0.067-0.878; $P=0.02$ ), with final model $R^{2}=0.549$ (Table 1 ), that confirmed the robustness of the association between the two mortality rates.

Life expectancy at birth, influenza vaccination coverage in the elderly (direct associations), number of hospital beds and CVD mortality rates (inverse associations) were significantly associated with both influenza and COVID-19 mortality rates, while population size was directly associated with COVID-19 mortality (Table 2).

The countries with population lower than 2000000 were Lichtenstein, Iceland, Malta, Luxembourg, Cyprus, Latvia and Estonia. The analyses repeated considering only the remaining 27 countries improved the association between influenza and COVID-19 mortality rates, and confirmed the previous results. Namely, Spearman correlation $\rho=0.476(P=0.01)$, simple regression coefficient $b=0.837$ (95\% confidence interval, $0.326-1.349$; $\left.P=0.002 ; R^{2}=0.313\right)$, multiple regression coefficient $b=0.887$ (95\% confidence interval, $0.438-1.336 ; P=0.0004 ; R^{2}=0.496$ ) (data not shown in table).

Ten countries were classified in the same COVID-19 and influenza mortality quartiles, with a fair absolute agreement of $29.4 \%$, that was higher for countries in the first and the highest quartiles. Namely, Bulgaria, Cyprus, Lichtenstein and Slovakia were in the first quartiles, and Belgium, France, Netherlands, Sweden were in the fourth quartiles (Supplementary Table S3). Seventeen countries showed a discrepancy of only one quartile, while two-quartile discrepancies were reported for seven countries and no third-quartile discrepancy was found. The ICC resulted 0.44 (95\% confidence interval, $0.12-0.68$ ).
Table 1. Simple and multiple regression analyses with crude COVID-19 mortality rate as dependent variable

\begin{tabular}{lccc}
\hline & Coefficient & $\begin{array}{c}95 \% \text { confidence } \\
\text { interval }\end{array}$ & $P$ \\
\hline Simple regression & & \\
\hline Influenza mortality & 0.743 & $0.272-1.214$ & 0.003 \\
\hline Multiple regression - initial model & & \\
\hline Influenza mortality & 0.561 & $0.140-0.982$ & 0.01 \\
\hline Population & 0.336 & $0.144-0.529$ & 0.001 \\
\hline Pneumonia mortality & 0.413 & $-0.200-1.027$ & 0.17 \\
\hline CVD mortality & -0.935 & -1.687 to -0.182 & 0.01 \\
\hline Multiple regression - final model & & 0.02 \\
\hline Influenza mortality & 0.472 & $0.067-0.878$ & 0.007 \\
\hline Population & 0.351 & $0.157-0.545$ & $<0.0001$ \\
\hline CVD mortality & -1.043 & -1.788 to -0.299 & \\
\hline
\end{tabular}

All variables were log transformed.

Influenza mortality, 3-year average crude influenza mortality rate; population, 3-year average population; pneumonia mortality, crude pneumonia mortality rate; CVD mortality, crude cardio-vascular disease mortality rate.

Simple regression $R^{2}=0.244$; multiple regression initial model $R^{2}=0.576$; final model $R^{2}=$ 0.549 .

The highest COVID-19 mortality rates estimated through multiple regression were provided for France, Germany, Spain, Italy and UK, while the lowest were provided for Lichtenstein, Slovakia, Hungary, Bulgaria and Cyprus (Supplementary Table S4). As expected, the multiple regression model provided higher agreement between quartile distributions. Indeed, the absolute agreement was $55.9 \%$ (19 countries) and ICC $=0.723$ ( $95 \%$ confidence interval, 0.512-0.852) (Supplementary Table S3).

\section{Discussion}

This analysis showed that 3-year average influenza mortality rate was associated with COVID-19 mortality rate in the European context, although influenza mortality alone could explain only part of the COVID-19 mortality variability. The discrepancy between the two mortality rates was likely due to the aforementioned differences between the two diseases at population and individual levels [26, 31].

An apparently perplexing characteristic of the reported association between the two mortality rates was that while influenza virus circulation during the seasons considered in the present analysis was uncontrolled, SARS-CoV-2 circulation was probably limited by the widespread exceptional public health measures implemented in Europe [32]. Therefore, assuming that the reported association between the two rates was not spurious, the most likely explanation of the present results was that SARS-CoV-2 circulation also was partly uncontrolled. Actually, surveys and phylogenetic analyses support the idea of multiple introductions of the virus in Europe since 2019 [10-15]. Such an undetected virus circulation is not surprising, since patients with COVID-19/CLI have been frequently misclassified as patients with ILI $[23,24]$, and is corroborated by SARS-CoV-2 seroprevalence surveys $[17-19,33]$. The implementation of country-based control policies likely prevented further severe SARS-CoV-2 outbreak propagation, thus explaining the COVID-19 incidence rate of $3-5 \%$ in May 2020, lower than 
Table 2. Associations between demographic, health and healthcare determinants and 3-year average crude influenza mortality rate and crude COVID-19 mortality rate (log transformed), assessed through simple regression analyses (regression coefficients; 95\% confidence intervals in brackets)

\begin{tabular}{|c|c|c|}
\hline Determinant & Influenza mortality rate & COVID-19 mortality rate \\
\hline 3-year average population $2014-2016^{a}$ & $0.05(-0.13$ to 0.24$)$ & $0.37(0.12-0.61)^{\mathrm{b}}$ \\
\hline Life expectancy at birth 2018 & $0.07(0.03-0.12)^{b}$ & $0.12(0.06-0.18)^{b}$ \\
\hline Healthy life expectancy at birth $2018^{c}$ & $0.01(-0.02$ to 0.03$)$ & $0.03(-0.01$ to 0.07$)$ \\
\hline 3-year average crude mortality rate $2014-2016^{a}$ & $-0.17(-1.31$ to 0.96$)$ & $-0.83(-2.50$ to 0.85$)$ \\
\hline 3-year average crude pneumonia mortality rate $2014-2016^{a}$ & $-0.38(-0.92$ to 0.16$)$ & $0.35(-0.49$ to 1.18$)$ \\
\hline Crude cardiovascular disease mortality rate $2016^{a}$ & $-0.64(-1.26 \text { to }-0.02)^{\mathrm{b}}$ & $-1.29(-2.18 \text { to }-0.41)^{\mathrm{b}}$ \\
\hline Hospital beds $\times 100000$ population $2017^{\text {a }}$ & $-0.70(-1.49 \text { to }-0.10)^{\mathrm{b}}$ & $-1.22(-2.40 \text { to }-0.04)^{b}$ \\
\hline 3-year average influenza vaccination population $\geq 65$ years $(2014-2016)^{d}$ & $0.01(0.002-0.01)^{b}$ & $0.02(0.01-0.02)^{b}$ \\
\hline
\end{tabular}

${ }^{a}$ Log transformed.

${ }^{\mathrm{b}} P<0.05$.

${ }^{\mathrm{C}}$ Available for 32 countries

${ }^{\mathrm{d}}$ Available for 31 countries.

influenza rate that is usually $10 \%$ or higher $[34,35]$. The history of other HCoVs corroborates this hypothesis. For example, the first patient infected with $\mathrm{HCoV}-\mathrm{NL63}$, a child with atypical bronchiolitis, was detected in January 2003 in Amsterdam. Soon after, HCoV-NL63 positive patients from all over the world with upper and/or lower respiratory tract infections were detected, and seroprevalence values as high as $2-9 \%$ were reported. Such an apparently rapidly spreading pandemic was explained by the long undetected virus circulation confirmed by the analysis of a specimen collected from a child with pneumonia that was stored on kidney simian cells since 1988. Thus, HCoV-NL63 was already circulating fifteen years before its detection $[16,36]$.

Relatively free SARS-CoV-2 circulation in Europe also was promoted by inefficient and untimely crisis coordination at central level [29], and by delays and contradictions of some international public health organisations in acknowledging community transmission, typical of pandemics, that must lead to public health control measures. Indeed, on 19 April 2020, community transmission was not acknowledged yet in France, Spain, UK, Italy, where 15000-25000 COVID-19 deaths were already reported, and in Belgium and the Netherlands, with 3500-5000 deaths, but was confirmed in small countries such as San Marino, Andorra, Bosnia and Kosovo [37]. The question remains unanswered, on whether earlier community transmission acknowledgement in Europe, and consequent timely implementation of coordinated COVID-19 control measures would have limited the high burden of COVID-19.

The current study corroborated the assumption that influenza and COVID-19 mortality rates share similar determinants. Indeed, both diseases were significantly associated with similar demographic, health and healthcare determinants, excluding population size that was associated only with COVID-19 (Table 2). This is also the reason why crude mortality rates were used instead of standardised rates, as the standardisation process would have reduced the impact of population age structure on mortality rates, while the rationale of the current study was that influenza and COVID-19 share similar population-based determinants, and population age structure was among them.

Influenza and COVID-19 share another important population-level characteristic. Namely, the problem of misclassifications and disagreements in classification that lead to inconsistent burden of disease estimates. Although influenza has been recognised as an important cause of mortality, particularly in the elderly, mortality rates are generally low, because much of related mortality is not attributed to primary influenza infection, but to complications and secondary infections. This problem generated incongruences in classifying influenza as underlying or contributing cause of death [38]. As for COVID-19, differences in mortality between countries and even within countries were partly attributable to the use of different criteria to classify COVID-19 deaths [39]. To overcome the problem of misclassification the US National Center for Health Statistics coined an aggregated variable called 'PIC', that considered all deaths attributed to pneumonia, influenza and COVID-19, updating another variable called 'P\&I', based on influenza and pneumonia [40]. In the current study, however, pneumonia mortality did not result associated with influenza and COVID-19 (Table 2), and unreported analyses using 3-year average 'P\&I' mortality rate provided non-significant results.

Influenza and COVID-19 mortality rates resulted associated with population age structure, as shown in Table 2, and corroborated by the EUROSTAT report showing that between 2012 and 2016 , as many as $70 \%$ influenza deaths occurred in the elderly aged $\geq 65$ years, and the European standardised influenza mortality rates in this age group were between ten and twenty times higher than in subjects younger than 65 years [41]. The COVID-19 burden in the elderly was even higher. Indeed, the elderly aged $\geq 65$ years accounted for $90-95 \%$ of deaths in European countries and their risk of dying was up to 80 times higher than in younger individuals [42]. Another characteristic shared by influenza and COVID-19 deaths was the impact of comorbidities on mortality. Indeed, three-fourth influenza deaths occur in patients with comorbidities [43], while for COVID-19 such a proportion is higher than $90 \%[42,44]$.

This study reported an inverse association between number of hospital beds and mortality rates (Table 2), thus showing that high influenza and COVID-19 mortality was also due to inefficiencies of the healthcare systems, and corroborated by data from several European countries [45]. Similarly, the inadequateness of the healthcare system has been responsible for the high COVID-19-related death toll reported in many countries, such as UK [5], Italy [46] and Spain [47].

The direct association between influenza vaccination coverage among the elderly and influenza and COVID-19 mortality rates reported in this study (Table 2) was corroborated by population- 
based studies, and the Cochrane systematic review focusing on the efficacy of influenza vaccination in the elderly showing an unclear effect on improving mortality [48-50]. This paradoxical effect of influenza vaccine is due to the fact that vaccine uptake is more likely in the categories who need it least, that is, women, elderly younger than 80 years and subjects without comorbidities [51], an effect called Inverse Care Law by Julian Tudor Hart in 1971, who stated that 'The availability of good medical care tends to vary inversely with the need for it in the population served' [52]. Unfortunately, the Inverse Care Law also applies to preventive medicine including cancer screening $[53,54]$, and influenza vaccination [55], and explains the apparently puzzling direct association between influenza vaccination coverage and COVID-19 mortality, since COVID-19 mortality risk was twofold higher in men than in females, 13-fold higher in individuals older than 80 years than in those aged 65-79 years, and 5-to-15-fold higher in patients with comorbidities, than in those without [56]. In other words, individuals at higher influenza and COVID-19 mortality risk are those who are less likely to get vaccinated. The reported association between high influenza vaccine coverage and high influenza and COVID-19 mortality has nothing to do with intrinsic vaccine efficacy, since ecologic studies are subject to ecologic fallacy that prevents from inferring associations at an individual level.

The multiple regression analysis showed that CVD mortality was inversely associated with COVID-19 mortality (Table 1). CVD, particularly ischaemic heart disease and stroke, is the leading cause of death in Europe, accounting for $40 \%$ and $49 \%$ of all deaths in males and females, respectively, and is also the leading cause of premature death, accounting for more than $35 \%$ of all deaths under 75 years. Differences in CVD mortality are, therefore, the main responsible of differences in life expectancy at birth, country distribution for age, and potential years of life lost. These differences are particularly evident between Eastern and Western European countries [57]. CVD and older age are also the main risk factors associated with COVID-19 death at an individual level [58-60].

These considerations help explain why Western European countries showed generally high influenza and COVID-19 mortality rates, while Eastern European countries showed the reverse. Indeed, COVID-19 and influenza are particularly lethal in elderly individuals and, thus, influenza and COVID-19 mortality rates are particularly high in countries where the proportion of elderly is higher. Since CVD deaths are responsible for premature mortality, countries where CVD mortality is higher, also are those with the lowest proportion of elderly and, consequently, with the lowest proportion of susceptible individuals at higher risk of dying from both COVID-19 and influenza.

Present research is an ecologic study with all the corresponding advantages and disadvantages of this approach. Indeed, the use of aggregated data prevented the identification of associations at an individual level, a problem known as ecologic fallacy. On the other hand, since these studies are relatively simple and reproducible they provide useful information in emergency situations like the COVID-19 pandemic. Such information, however, must be considered carefully and implementing public health control measures on the basis of ecologic studies alone could be problematic [61]. During this pandemic several ecologic studies have been published, reporting associations between COVID-19 incidence and mortality rates and BCG vaccine coverage [62], malaria prevalence [63], environmental and meteorological factors, pollutants (reviewed in [64]), etc. Although these associations were robust enough, they could not be considered to design COVID-19 control policies, due to ecologic fallacy. In the same way, the current study did not show that influenza prevention at an individual level leads to COVID-19 prevention, but only that the two mortality rates were associated at the population level.

The second important limit of this study was the reported problem of the reliability of both influenza and COVID-19 death counts $[38,39]$, that could lead to uncertainties in the true mortality rates in the countries under investigation in this study. An ideal, yet unfeasible, approach would be that every dying individual with ILI, CLI, acute respiratory illness and pneumonia was tested for all the circulating influenza strains and for SARS-CoV-2. In the case of influenza, this uncertainty regarding the death counts, led to varying estimates of the global number of attributable deaths, ranging from the lowest limit provided by the Global Burden of Disease Study of 99000 , to the highest limit provided by the CDC of 650000 [65]. The problem of consistency of aggregated data, however, is shared by almost all diseases and conditions. For example, the estimated global number of deaths from breast cancer was 630000 according to GLOBOCAN 2018 [66], and 535000 according to the Global Burden of Disease Study [67], with important differences within each country.

The last limitation of this study was that influenza mortality rate alone could not be considered an optimal COVID-19 mortality rate predictor, since the multiple regression analysis showed that there were other important population-based confounders associated with COVID-19 mortality. They could be variables related to age structure and prevalence of comorbidities associated with COVID-19 mortality. For example, age structure explained part of the between-country differences in COVID-19 mortality and case-fatality rates $[4,20]$; median prevalence of the five conditions most frequently associated with severe COVID-19 in USA allowed to identify the areas at highest risk for COVID-19 death [21]; age-specific prevalence of comorbidities explained the differences in mortality between Nigeria, Brazil and Italy [22]. Economic and healthcare associated variables are other aggregated data potentially useful to predict COVID-19 severity and spread [68-70], as well as inequalities within the general population [71]. Unlike these studies, however, the present analysis considered the mortality rate from an infectious disease that was not somewhat causally associated with COVID-19 mortality and death and was based on a different assumption, namely, that the two diseases shared a set of determinants, ranging from the characteristics of the population at highest risk, to transmission routes, from case and death misclassifications, to the efficiency of the healthcare systems.

In conclusion, influenza and COVID-19 mortality rates were significantly associated and influenza mortality could be an eligible predictor for the design of more accurate multivariable COVID-19 mortality assessment and prediction models.

Supplementary material. The supplementary material for this article can be found at https://doi.org/10.1017/S0950268820002125

Conflict of interest. None.

Data availability statement. The data that support the findings of this study can be downloaded from the ECDC and EUROSTAT databases and are, in part, displayed in Supplementary material.

\section{References}

1. World Health Organization (2020) Coronavirus Disease 2019 (COVID-19) Situation Report - 51. Geneva, Switzerland: World Health Organization. 
Available at https://www.who.int/docs/default-source/coronaviruse/situationreports/20200311-sitrep-51-covid-19.pdf?sfvrsn=1ba62e57_10.

2. European Centre for Disease Prevention and Control. COVID-19 database. Available at https://www.ecdc.europa.eu/en/covid-19-pandemic (Accessed 31 May 2020).

3. Gibney E (2020) Whose coronavirus strategy worked best? Scientists hunt most effective policies. Nature 581, 15-16.

4. Dowd JB et al. (2020) Demographic science aids in understanding the spread and fatality rates of COVID-19. Proceedings of the National Academy of Science USA 117, 9696-9698.

5. Griffin S (2020) COVID-19: 'staggering number' of extra deaths in community is not explained by COVID-19. BMJ 369, m1931.

6. Huber K and Goldstein P (2020) COVID-19: implications for prehospital, emergency and hospital care in patients with acute coronary syndromes. European Heart Journal. Acute Cardiovascular Care 9, 204-208.

7. Verelst F, Kuylen E and Beutels P (2020) Indications for healthcare surge capacity in European countries facing an exponential increase in coronavirus disease (COVID-19) cases, March 2020. Euro Surveillance 25, 2000323.

8. CDC COVID-19 Response Team (2020) Geographic differences in COVID-19 cases, deaths, and incidence - United States, February 12April 7, 2020. MMWR Morbidity Mortality Weekly Report 69, 465-471.

9. Grasselli G, Pesenti A and Cecconi M (2020) Critical care utilization for the COVID-19 outbreak in Lombardy, Italy: early experience and forecast during an emergency response. JAMA 323, 1545-1546.

10. Lavezzo E et al. (2020) Suppression of a SARS-CoV-2 outbreak in the Italian municipality of Vo. Nature 584, 425-429.

11. Valenti $\mathrm{L}$ et al. SARS-CoV-2 seroprevalence trends in healthy blood donors during the COVID-19 Milan outbreak. Preprint at: medRxiv. 2020.05.11.20098442. doi: 10.1101/2020.05.11.20098442.

12. Deslandes A et al. (2020) SARS-CoV-2 was already spreading in France in late December 2019. International Journal of Antimicrobial Agents 55, 106006.

13. Stefanelli P et al. (2020) Whole genome and phylogenetic analysis of two SARS-CoV-2 strains isolated in Italy in January and February 2020: additional clues on multiple introductions and further circulation in Europe. Euro Surveillance 25, 2000305.

14. van Dorp L et al. (2020) Emergence of genomic diversity and recurrent mutations in SARS-CoV-2. Infection Genetics and Evolution 83, 104351.

15. Zehender G et al. (2020) Genomic characterization and phylogenetic analysis of SARS-COV-2 in Italy. Journal of Medical Virology 92, 1637-1640.

16. van der Hoek L, Pyrc K and Berkhout B (2006) Human coronavirus NL63, a new respiratory virus. FEMS Microbiology Reviews 30, 760-773.

17. Folkhalsomyndigheten database. Available at https://www.folkhalsomyndigheten.se/nyheter-och-press/nyhetsarkiv/2020/maj/forsta-resultaten-franpagaende-undersokning-av-antikroppar-for-covid-19-virus/ (Accessed 25 May 2020).

18. Institudo de Salud Carlos III database. Available at https://www.isciii.es/ Noticias/Noticias/Paginas/Noticias/PrimerosDatosEstudioENECOVID19. aspx?fbclid=IwAR0askXAC4Is4rSY8EHR6S9TYAMZQWh6mAvuHNyUj NgiZP3TWe6Sj3pcwGo (Accessed 25 May 2020).

19. Ioannidis JPA. The infection fatality rate of COVID-19 inferred from seroprevalence data. Preprint at: medRxiv. 2020.05.13.20101253. doi: 10.1101/2020.05.13.20101253.

20. Sudharsanan $\mathbf{N}$ et al. (2020) The contribution of the age distribution of cases to COVID-19 case fatality across countries: a 9-country demographic study. Annals of Internal Medicine. Published online 22 July 2020. doi: 10.7326/M20-2973.

21. Razzaghi H et al. (2020) Estimated county-level prevalence of selected underlying medical conditions associated with increased risk for severe COVID-19 illness - United States, 2018. MMWR. Morbidity and Mortality Weekly Report 69, 945-950.

22. Nepomuceno MR et al. (2020) Besides population age structure, health and other demographic factors can contribute to understanding the COVID-19 burden. Proceedings of the National Academy of Sciences USA 117, 13881-13883.

23. Centers for Disease Control and Prevention. COVIDView database. Available at https://www.cdc.gov/coronavirus/2019-ncov/covid-data/covidview/index.html (Accessed 29 May 2020).
24. Boëlle PY et al. (2020) Excess cases of influenza-like illnesses synchronous with coronavirus disease (COVID-19) epidemic, France, March 2020. Euro Surveillance 25, 2000326.

25. Sakamoto $\mathbf{H}$, Ishikane $\mathbf{M}$ and Ueda $\mathbf{P}$ (2020) Seasonal influenza activity during the SARS-CoV-2 outbreak in Japan. JAMA 323, 1969-1971.

26. Faust JS and Del Rio C (2020) Assessment of deaths from COVID-19 and from seasonal influenza. JAMA Internal Medicine 180, 1045-1046.

27. Tolksdorf K et al. (2020) Influenza-associated pneumonia as reference to assess seriousness of coronavirus disease (COVID-19). Euro Surveillance 25, 2000258.

28. EUROSTAT database. Available at https://ec.europa.eu/eurostat/data/ database (Accessed 31 May 2020).

29. European Commission. Coronavirus response. Public Health. Crisis Coordination. Available at https://ec.europa.eu/info/live-work-travel-eu/ health/coronavirus-response/public-health_en\#crisis-coordination (Accessed 10 July 2020).

30. McGraw KO and Wong SP (1996) Forming inferences about some intraclass correlation coefficients. Psychological Methods 1, 30-46.

31. Lockerd Maragakis L. Coronavirus Disease 2019 vs. the Flu. Johns Hopkins Medicine. Health. Available at https://www.hopkinsmedicine. org/health/conditions-and-diseases/coronavirus/coronavirus-disease-2019-vsthe-flu (Updated 1 June 2020).

32. Vokó Z and Pitter JG (2020) The effect of social distance measures on COVID-19 epidemics in Europe: an interrupted time series analysis. Geroscience 42, 1075-1082.

33. Percivalle E et al. (2020) Prevalence of SARS-CoV-2 specific neutralising antibodies in blood donors from the Lodi Red Zone in Lombardy, Italy, as at 06 April 2020. Eurosurveillance 25, 2001031.

34. Jayasundara K et al. (2014) Natural attack rate of influenza in unvaccinated children and adults: a meta-regression analysis. BMC Infectious Diseases 14, 670 .

35. Tokars JI, Olsen SJ and Reed C (2018) Seasonal incidence of symptomatic influenza in the United States. Clinical Infectious Diseases 66, 15111518.

36. Fouchier RA et al. (2004) A previously undescribed coronavirus associated with respiratory disease in humans. Proceedings of the National Academy of Science USA 101, 6212-6216.

37. World Health Organization. Coronavirus Disease 2019 (COVID-19). Situation Report 90.19 April 2020. Available at https://www.who.int/ docs/default-source/coronaviruse/situation-reports/20200419-sitrep-90covid-19.pdf?sfvrsn=551d47fd_2 (Accessed 10 July 2020).

38. Gordon A and Reingold A (2018) The burden of influenza: a Complex problem. Current Epidemiology Reports 5, 1-9.

39. COVID-19 Health System Response Monitor. How comparable is COVID-19 mortality across countries? 4 June 2020. Available at https:// analysis.covid19healthsystem.org/index.php/2020/06/04/how-comparableis-covid-19-mortality-across-countries/ (Accessed 6 July 2020).

40. Centers for Disease Control and Prevention. National Center for Health Statistics. Technical Notes. Provisional Death Counts for Coronavirus Disease (COVID-19) 13 May 2020. Available at https://www.cdc.gov/ nchs/nvss/vsrr/covid19/tech_notes.htm (Accessed 6 July 2020).

41. EUROSTAT. How many EU citizens die from influenza? 7 April 2020. Available at https://ec.europa.eu/eurostat/web/products-eurostat-news/-/ DDN-20200407-1 (Accessed 31 May 2020).

42. Ioannidis JPA, Axfors C and Contopoulos-Ioannidis DG (2020) Population-level COVID-19 mortality risk for non-elderly individuals overall and for non-elderly individuals without underlying diseases in pandemic epicenters. Environ Research 188, 109890.

43. Cromer D et al. (2014) The burden of influenza in England by age and clinical risk group: a statistical analysis to inform vaccine policy. Journal of Infection 68, 363-371.

44. European Centre for Disease Prevention and Control (2020) Coronavirus Disease 2019 (COVID-19) in the EU/EEA and the UK Ninth Update, 23 April 2020. Stockholm: ECDC.

45. Federici C et al. (2018) Health care costs of influenza-related episodes in high income countries: a systematic review. PLoS One 13, e0202787.

46. Armocida B et al. (2020) The Italian health system and the COVID-19 challenge. Lancet Public Health 5, e253. 
47. Legido-Quigley H et al. (2020) The resilience of the Spanish health system against the COVID-19 pandemic. Lancet Public Health 5, e251-e252.

48. Anderson ML, Dobkin C and Gorry D (2020) The effect of influenza vaccination for the elderly on hospitalization and mortality: an observational study with a regression discontinuity design. Annals of Internal Medicine 172, 445-452.

49. Demicheli V et al. (2018) Vaccines for preventing influenza in the elderly. Cochrane Database of Systematic Reviews 2, CD004876.

50. Rizzo C et al. (2006) Influenza-related mortality in the Italian elderly: no decline associated with increasing vaccination coverage. Vaccine 24, 6468-6475.

51. Fireman B et al. (2009) Influenza vaccination and mortality: differentiating vaccine effects from bias. American Journal of Epidemiology 170, 650-656.

52. Hart JT (1971) The inverse care law. Lancet 1, 405-412.

53. Petti S and Scully C (2015) How many individuals must be screened to reduce oral cancer mortality rate in the western context? A challenge. Oral Diseases 21, 949-954.

54. Wiener RS and Rivera MP (2019) Access to lung cancer screening programs in the United States: perpetuating the inverse care law. Chest 155, 883-885.

55. Story A et al. (2014) Influenza vaccination, inverse care and homelessness: cross-sectional survey of eligibility and uptake during the 2011/12 season in London. BMC Public Health 14, 44.

56. The OpenSAFELY Collaborative et al. OpenSAFELY: factors associated with COVID-19-related hospital death in the linked electronic health records of 17 million adult NHS patients. Preprint at: medRxiv. 2020.05.06.20092999. doi: 10.1101/2020.05.06.20092999.

57. Wilkins E et al. (2017) European Cardiovascular Disease Statistics 2017. Brussels: European Heart Network.

58. Grasselli G et al. (2020) Risk factors associated with mortality among patients with COVID-19 in intensive care units in Lombardy, Italy. JAMA Internal Medicine. Published online 15 July 2020. doi: 10.1001/ jamainternmed.2020.3539.

59. Gupta $\mathrm{S}$ et al. (2020) Factors associated with death in critically ill patients with coronavirus disease 2019 in the US. JAMA Internal Medicine. Published online 15 July 2020. doi: 10.1001/jamainternmed.2020.3596.
60. Sun YJ et al. (2020) Clinical features of fatalities in patients with COVID-19. Disaster Medicine and Public Health Preparedness. Published online 15 July 2020. doi: 10.1017/dmp.2020.235.

61. Wakefield J (2008) Ecologic studies revisited. Annual Reviews of Public Health 29, 75-90.

62. Escobar LE et al. (2020) BCG Vaccine protection from severe coronavirus disease 2019 (COVID-19). Proceedings of the National Academy of Science USA 117, 17720-17726.

63. Napoli PE and Nioi M (2020) Global spread of coronavirus disease 2019 and malaria: an epidemiological paradox in the early stage of a pandemic. Journal of Clinical Medicine 9, 1138.

64. Shakil MH et al. (2020) COVID-19 and the environment: a critical review and research agenda. Science of the Total Environment 745, 141022.

65. Paget J et al. (2019) Global mortality associated with seasonal influenza epidemics: new burden estimates and predictors from the GLaMOR Project. Journal of Global Health 9, 020421.

66. International Agency for Research on Cancer. Cancer Today database. Available at https://gco.iarc.fr/today/home (Accessed 25 July 2020).

67. Global Burden of Disease Cancer Collaboration et al. (2018) Global, regional, and national cancer incidence, mortality, years of life lost, years lived with disability, and disability-adjusted life-years for 29 cancer groups, 1990 to 2016: a systematic analysis for the global burden of disease study. JAMA Oncology 4, 1553-1568.

68. Ji Y et al. (2020) Potential association between COVID-19 mortality and health-care resource availability. Lancet Global Health 8, e480.

69. Rafael RMR et al. (2020) Effect of income on the cumulative incidence of COVID-19: an ecological study. Revista Latino-Americana de Enfermagem 28, e3344.

70. Lippi G et al. (2020) The death rate for COVID-19 is positively associated with gross domestic products. Acta Biomedica 91, 224-225.

71. Oronce CIA, et al. (2020) Association between state-level income inequality and COVID-19 cases and mortality in the USA. Journal of General Internal Medicine 35, 2791-2793. 